\title{
Numerical Simulation for Temperature Field Evolution of Metallic Alloy Surface due to Pulsed Laser Treatment
}

\section{Youshi HONG, Zhongsheng AN and Huicai WANG}

\author{
Stete Key Laboratory of Non-linear Mechanics (LNM), \\ Institute of Mechanics, Chinese Academy of Sciences, Beijing 100080, China
}

Keywords: Pulsed Nd:YAG laser, Stefan problem, enthalpy method, temperature field, latent heat

\begin{abstract}
This paper performed a numerical simulation on temperature field evolution for the surface layer of a metallic alloy subjected to pulsed Nd:YAG laser treatment. The enthalpy method was adopted to solve the moving boundary problem, i.e. Stefan problem. Computational results were obtained to show the temperature field evolution. Effects of latent heat and mushy zone width on the temperature field were investigated. The results also show very high values of temperature gradient and cooling rate, which are typical characteristics during the solidification process.
\end{abstract}

\section{Introduction}

The technique of laser treatment on material surface so as to improve mechanical properties of the surface layer has been rapidly developed during the previous decades. The surface texturing method used pulsed Nd:YAG laser has been successfully created for the treatment of the cold-roll used in steel sheet forming [1,2]. To understand the mechanism of interaction between Nd:YAG laser and metallic material surface becomes essential for the further exploring the capacity of such a promising technique. Thus, this paper performs a numerical research on the melting/solidification process of metallic alloy surface exposed by pulsed Nd:YAG laser irradiation. The movement of solid/liquid interface is considered. The moving boundary problem is called Stefan problem due to the early work by Stefan on ice/water mixture [3,4]. We establish a two-phase moving boundary model to calculate the temperature field and to describe the melting/solidification process caused by the pulsed laser treatment. The results show the very high values of temperature gradient and cooling rate during the solidification process. The effects of latent heat and mushy zone width on the temperature field are also discussed.

\section{Basic Model}

Being irradiated by high energy density pulsed laser, the metallic specimen surface will absorb a portion of laser energy, which will lead to the temperature rising and produce temperature gradient from surface to substrate. When the temperature of local surface rises to the melting point, a small liquid pool will form. For metallic alloys, a mushy zone bounded with the liquid pool will appear, where liquid and solid phases co-exist. The interface of liquid/mushy zone or solid/mushy zone moves with the heat conduction process. For this case, we develop a physical model as schematically shown in Fig.1 to describe the temperature field. The laser beam is considered as circular shape and a 2-D axisymmetrical coordinate system is constructed (Fig.2). For the Nd:YAG laser, the pulse time is several micro-seconds and the beam radius is tens of microns. In order to simulate such an extraordinary problem of high energy density, short time duration and small spatial dimension, we make the following assumptions: (1) the absorption of laser energy happens in a thin surface layer of specimen and the laser energy input is treated as a surface heat source; (2) the energy density of laser beam is of Gaussian distribution and the central point on the surface has the peak value of energy density; (3) the heat transfer due to fluid flow in the pool is neglected; (4) the heat affected region due to laser irradiation is far less than the size of the whole specimen; and (5) the surface of the molten liquid zone is flat. 
Laser beam

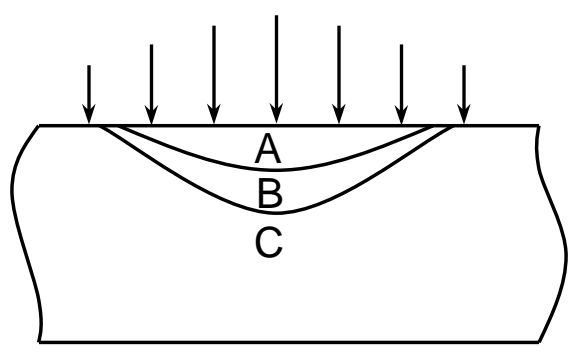

Fig.1 Schematic of surface profile due to laser beam irra- Fig.2 Axisymmetrical coordinate system. diation. (A: liquid zone; B: mushy zone; C: solid zone)
Laser beam

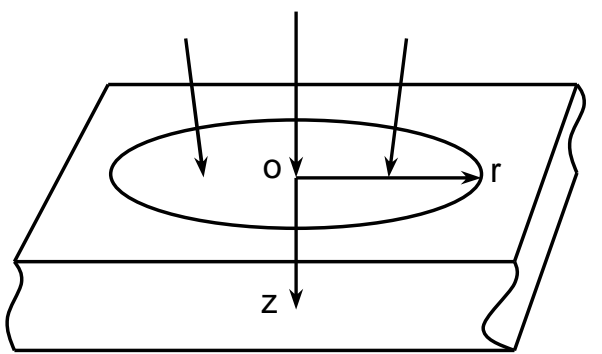

With above assumptions, we can apply Fourier conduction law in liquid zone, solid zone and mushy zone, respectively, and the governing equations in the coordinate system are established:

$$
\begin{array}{ll}
\frac{1}{r} \frac{\partial}{\partial r}\left(r \frac{\partial T^{1}}{\partial r}\right)+\frac{\partial^{2} T^{1}}{\partial z^{2}}-\frac{1}{a_{1}} \frac{\partial T^{1}}{\partial t}=0 & \text { for } g^{1}(\xi, \eta, t) \geq 0 \text { i.e. } T^{1} \geq T_{\mathrm{L}}, \\
\frac{1}{r} \frac{\partial}{\partial r}\left(r \frac{\partial T^{\mathrm{s}}}{\partial r}\right)+\frac{\partial^{2} T^{\mathrm{s}}}{\partial z^{2}}-\frac{1}{a_{\mathrm{s}}} \frac{\partial T^{\mathrm{s}}}{\partial t}=0 & \text { for } g^{\mathrm{s}}(\xi, \eta, t) \leq 0 \text { i.e. } T^{\mathrm{s}} \leq T_{\mathrm{S}}, \\
\frac{1}{r} \frac{\partial}{\partial r}\left(r \frac{\partial T^{\mathrm{m}}}{\partial r}\right)+\frac{\partial^{2} T^{\mathrm{m}}}{\partial z^{2}}-\frac{\rho}{K_{\mathrm{m}}} \frac{\partial Q\left(T^{\mathrm{m}}\right)}{\partial t}-\frac{1}{a_{\mathrm{m}}} \frac{\partial T^{\mathrm{m}}}{\partial t}=0 & \text { for } g^{\mathrm{l}}(\xi, \eta, t) \leq 0, g^{\mathrm{s}}(\xi, \eta, t) \geq 0 \text { i.e. } T_{\mathrm{S}} \leq T^{\mathrm{m}} \leq T_{\mathrm{L}},
\end{array}
$$

where $T$ and $t$ are temperature and time variables, $T_{\mathrm{S}}$ is solidus temperature, $T_{\mathrm{L}}$ is liquidus temperature, $a$ is thermal diffusivity, $K$ is thermal conductivity, $Q$ is latent heat of fusion, $\rho$ is mass density, and $r$ and $z$ are coordinates referring to Fig.2. Note that $g^{1}(\xi, \eta, t)=0$ and $g^{\mathrm{s}}(\xi, \eta, t)=0$ are two moving boundaries for the interfaces of liquid/mushy zone and mushy/solid zone as A/B and $\mathrm{B} / \mathrm{C}$ in Fig.1. The letters $1, \mathrm{~s}$ and $\mathrm{m}$ of superscript and subscript denote the value in liquid, solid and mushy zone, respectively. The fixed boundary conditions are:

$$
\begin{aligned}
& \frac{\partial T^{1}(r, 0, t)}{\partial z}=-\frac{q(r)}{K_{\mathrm{s}}}, \\
& \frac{\partial T^{\mathrm{s}}(\infty, z, t)}{\partial r}=\frac{\partial T^{\mathrm{s}}(r, \infty, t)}{\partial z}=0 \quad \text { and } \quad T^{\mathrm{s}}(\infty, z, t)=T^{\mathrm{s}}(r, \infty, t)=T_{0},
\end{aligned}
$$

where $q(r)$ is the laser energy density on the surface, representing energy input and $T_{0}$ is original temperature. The initial conditions are:

$$
T^{\mathrm{s}}(0,0,0)=T_{\mathrm{S}}, \quad T^{\mathrm{s}}(r, z, 0)=f(r, z) \quad \text { and } \quad g^{\mathrm{s}}(r, 0,0)=0,
$$

in which $f(r, z)$ is the temperature distribution at the time the molten pool is just produced, i.e. the central point of beam spot is at the solidus temperature $T_{\mathrm{S}}$. On the boundaries of mushy zone, the following conditions should be satisfied:

$$
\begin{aligned}
& K_{\mathrm{m}} \frac{\partial T^{\mathrm{m}}\left(\xi^{1}, \eta^{1}, t\right)}{\partial n}-K_{1} \frac{\partial T^{1}\left(\xi^{1}, \eta^{1}, t\right)}{\partial n}=Q v_{n}\left(\xi^{1}, \eta^{1}, t\right) \rho, \\
& K_{\mathrm{m}} \frac{\partial T^{\mathrm{m}}\left(\xi^{\mathrm{s}}, \eta^{\mathrm{s}}, t\right)}{\partial n}-K_{\mathrm{s}} \frac{\partial T^{\mathrm{s}}\left(\xi^{\mathrm{s}}, \eta^{\mathrm{s}}, t\right)}{\partial n}=0, \\
& T^{1}\left(\xi^{1}, \eta^{1}, t\right)=T_{\mathrm{L}} \quad \text { and } \quad T^{\mathrm{s}}\left(\xi^{\mathrm{s}}, \eta^{\mathrm{s}}, t\right)=T_{\mathrm{S}},
\end{aligned}
$$


where $v_{n}\left(\xi^{1}, \eta^{1}, t\right)$ is the mass velocity normal to the moving boundary $g^{1}\left(\xi^{1}, \eta^{1}, t\right)=0$. Because of the coupling between governing equations and moving boundaries, the solution to the problem becomes very complicated. The non-linearity of temperature gradient jump causes more difficulties in the numerical scheme of the problem. Note that the starting point of time is the instant when melting begins, so the conductivity problem has to be solved to acquire initial condition of $f(r, z)$.

\section{Method of Solution}

Several methods have been developed to solve the Stefan problem, or moving boundary problem, mainly making efforts on numerical solutions [4,5]. Typical numerical methods include Method of Lines, Variational Inequalities Method, Isotherm Migration Method and Enthalpy Method [4,6-8]. With regard to the present problem, pulsed laser irradiation with high energy density results in steep temperature gradient in the surface layer. For this two-phase multi-dimensional problem with a mushy zone, enthalpy method is an appropriate approach. We define the expression for enthalpy as

$$
H(T)= \begin{cases}\int_{T^{*}-\varepsilon}^{T} \rho(\theta) c_{\mathrm{s}}(\theta) \mathrm{d} \theta & \text { for } T \leq T^{*}-\varepsilon \\ \frac{\rho Q}{2 \varepsilon}\left(T-T^{*}+\varepsilon\right) & \text { for } T^{*}-\varepsilon \leq T \leq T^{*}+\varepsilon \\ \rho Q+\int_{T^{*}+\varepsilon}^{T} \rho(\theta) c_{1}(\theta) \mathrm{d} \theta & \text { for } T \geq T^{*}+\varepsilon\end{cases}
$$

where $c$ is specific heat, $T^{*}=T_{\mathrm{S}}+\left(T_{\mathrm{L}}-T_{\mathrm{S}}\right) / 2$ and $\varepsilon=\left(T_{\mathrm{L}}-T_{\mathrm{S}}\right) / 2(\varepsilon$ is the half width of mushy zone). The Kirchhoff transformation $\left[v=\int^{T} K(\zeta) \mathrm{d} \zeta\right]$ is adopted in which $K(\zeta)$ is the general expression of thermal conductivity. Then the problem described by Eqs.1-5 can be concisely expressed as:

$$
\begin{aligned}
& \frac{\partial H}{\partial t}=\frac{1}{r} \frac{\partial}{\partial r}\left(r \frac{\partial v}{\partial r}\right)+\frac{\partial^{2} v}{\partial z^{2}} \\
& \frac{\partial v(r, 0, t)}{\partial z}=q(r) \\
& \frac{\partial v(\infty, z, t)}{\partial r}=\frac{\partial v(r, \infty, t)}{\partial z}=0 \\
& v(\infty, z, t)=v(r, \infty, t)=v(r, z, 0)=0
\end{aligned}
$$

In the new description, the moving boundary conditions (Eq.5) are no longer obviously present. Therefore, when calculating $H$ and $v$ fields, we need not to distinguish computational region whether it is in solid zone, mushy zone or liquid zone. Based on this new description, an explicit calculation scheme can be executed as following steps: (a) at time step $n+1$, calculate $H$ field according to discretized Eq.7 from $v$ field of previous time $n$, (b) calculate new $v$ field at time step $n+1$ from $H$ field by Eq.6, and (c) calculate $v$ at boundaries according to Eqs.9 and 10. When above calculations in each time step are finished, based on Eq.6, positions of every phase zone can be determined and temperature field can be readily obtained.

\section{Calculation Results and Discussion}

A stainless steel is chosen as an example for calculation. The thermo-physical properties used for calculation are cited from Ref. [9] and [10] with $a=1.31 \times 10^{-5} \mathrm{~m}^{2} / \mathrm{s}, T_{\mathrm{S}}=1770 \mathrm{~K}, T_{\mathrm{L}}=1800 \mathrm{~K}$, $T_{\mathrm{V}}=3700 \mathrm{~K}\left(T_{\mathrm{V}}\right.$ : vaporization temperature $)$ and $Q=2.9 \times 10^{5} \mathrm{~J} / \mathrm{kg}$. The Nd:YAG laser has a continuous power of $200 \mathrm{~W}$ with a beam radius $R_{0}=70 \mu \mathrm{m}$. 
Temperature Field Evolution. Figure 3 shows the temperature evolution when the specimen of stainless steel is irradiated by pulsed Nd:YAG laser. It is shown that the temperature of alloy surface will rise to a certain level quickly. If we define the ratio of maximum depth of an isothermal contour to its diameter on the surface as depth/width ratio or simply called aspect ratio, then we can see aspect ratio of isotherms increases with time development, i.e. isotherms develop faster along depth direction than in radial one.
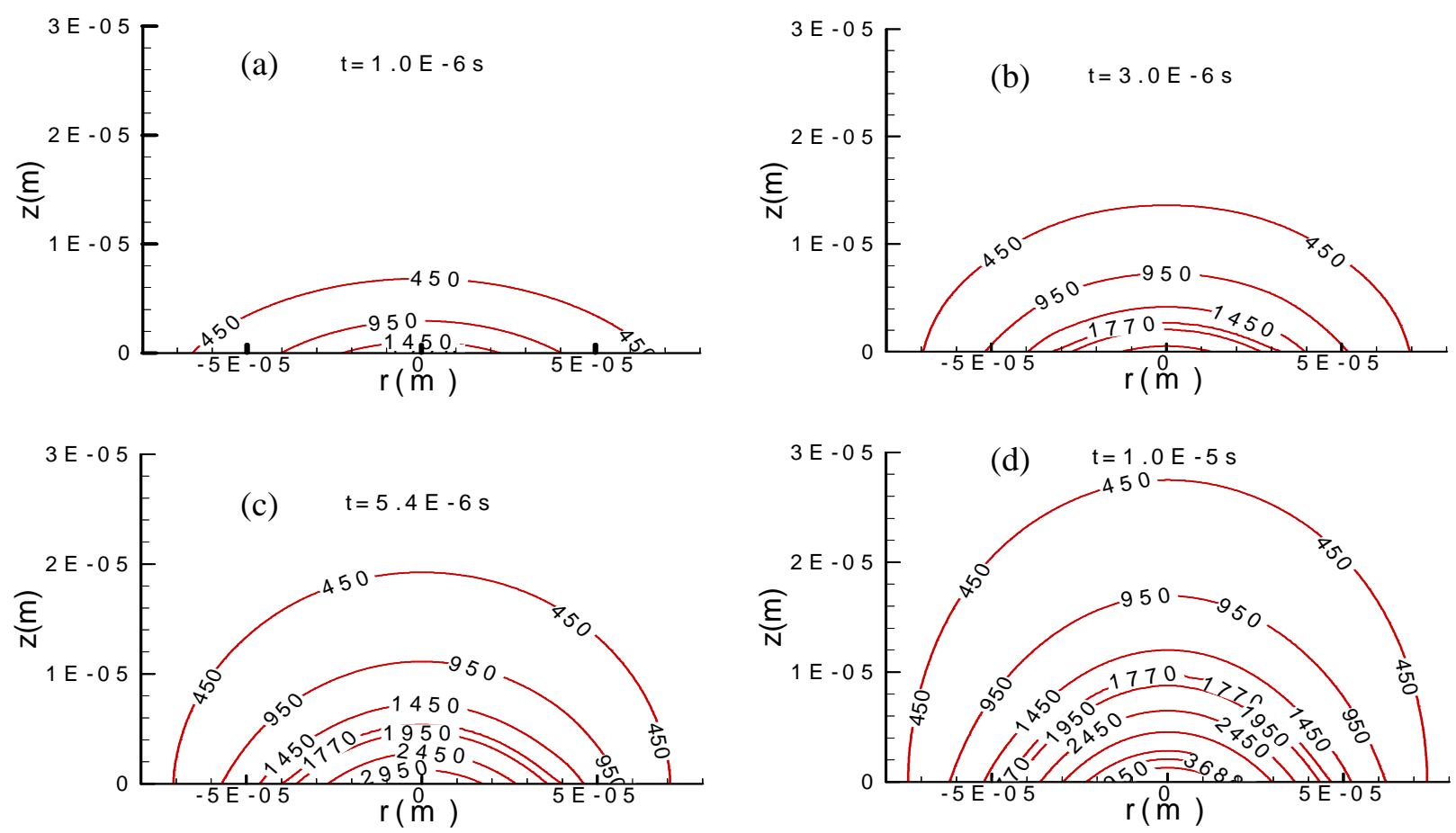

Fig.3 Evolution of temperature field from $1 \mu$ s to $10 \mu$ s after pulsed laser irradiation.

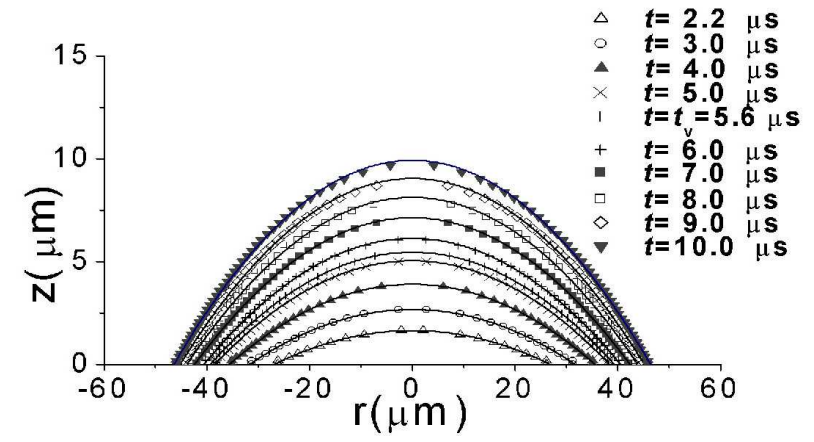

Fig.4 Evolution of molten zone from $2.2 \mu$ s to $10 \mu$ s after pulsed laser irradiation.

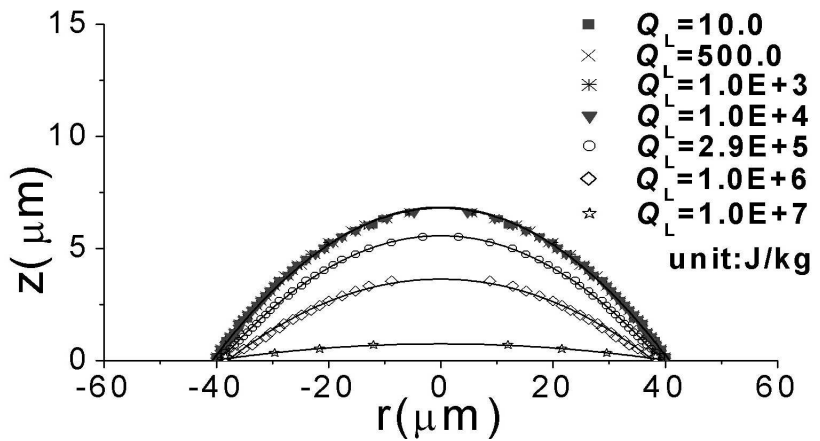

Fig.5 Shapes of molten zone for different values of $Q$ at $5.5 \mu$ s after pulsed laser irradiation.

Evolution of Moving Boundary. Figure 4 illustrates the temporal evolution of molten pool, from which we observe that the aspect ratio of the pool increases gradually. From the moment from $3 \mu \mathrm{s}$ to $7 \mu \mathrm{s}$, the aspect ratio changes from 0.041 to 0.082 . This is helpful to understand the influence of laser-texturing processing parameters on the roughness of specimen surface.

Effect of Latent Heat. The advantage of Stefan model is possible to study the effect of the latent heat of fusion. As shown in Fig.5, for cases in which latent heat $Q$ is less than the practical value of $2.9 \times 10^{5} \mathrm{~J} / \mathrm{kg}$ (i.e. $10,000,1,000,500$ and $10 \mathrm{~J} / \mathrm{kg}$ ), in general, the results at $t=5.5 \mu$ s have little difference. However, the difference between $Q=10.0 \mathrm{~J} / \mathrm{kg}$ and $Q=2.9 \times 10^{5} \mathrm{~J} / \mathrm{kg}$ is obvious, with the change of aspect ratio being nearly $19 \%$. While for the time when central point begins to evaporate, 
the difference between cases of $Q=10.0 \mathrm{~J} / \mathrm{kg}$ and $Q=2.9 \times 10^{5} \mathrm{~J} / \mathrm{kg}$ is diminished (Fig.6). Referring to the data in Table 1, the influence of $Q$ on vaporization time is relatively large (15.3\% in difference). Noting that all the vaporization time $t_{\mathrm{v}}$ is larger than $5.5 \mu \mathrm{s}$. We can conclude that latent heat will make the aspect ratio decreases to some extent, and it also causes vaporization time elongated. In addition, the width of molten pool almost holds stable, only the depth decreases with the increasing value of $Q$. This is understandable because the latent heat of fusion exists in mushy zone, where the material absorbs heat to complete phase change. The latent heat becomes the barrier of transmitting heat into solid zone. On the surface, temperature gradient is determined by heat flow boundary so that the barrier effects only in the radial direction. This leads to the fact that the depth direction is relatively affected by the latent heat. If laser pulse is terminated immediately after vaporization begins, then latent heat has very limited effect on the pulsed laser texturing processing. However, it is worth to notify that the latent heat of magnitude about $10^{5} \mathrm{~J} / \mathrm{kg}$ is in a sensitive range. Whether it can be neglected needs a further estimation by considering practical material and processing parameters.

Table. $1 t_{\mathrm{m}}, t_{\mathrm{v}}$ and aspect ratio of molten zone for different values of $Q$

\begin{tabular}{lccccccc}
\hline latent heat $Q(\mathrm{~J} / \mathrm{kg})$ & 10.0 & 500.0 & $1.0 \times 10^{3}$ & $1.0 \times 10^{4}$ & $2.9 \times 10^{5}$ & $1.0 \times 10^{6}$ & $1.0 \times 10^{7}$ \\
\hline melting time $t_{\mathrm{m}}(\mu \mathrm{s})$ & 0.979 & 0.979 & 0.979 & 0.979 & 0.979 & 0.979 & 0.979 \\
vaporization time $t_{\mathrm{v}}(\mu \mathrm{s})$ & 5.562 & 5.564 & 5.565 & 5.597 & 6.566 & 8.853 & $>10$ \\
$\quad$ aspect ratio at $t_{\mathrm{v}}$ & 0.08303 & 0.08302 & 0.08301 & 0.08289 & 0.07863 & 0.07122 & $0.01751^{*}$ \\
\hline$*=10 \mu \mathrm{s}$ & & & & & & &
\end{tabular}

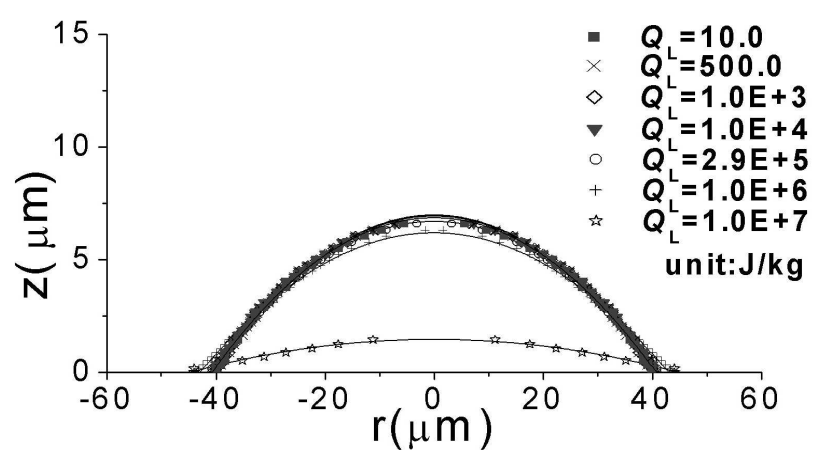

Fig.6 Shapes of molten zone for different values of $Q$ when central point begins to evaporate.

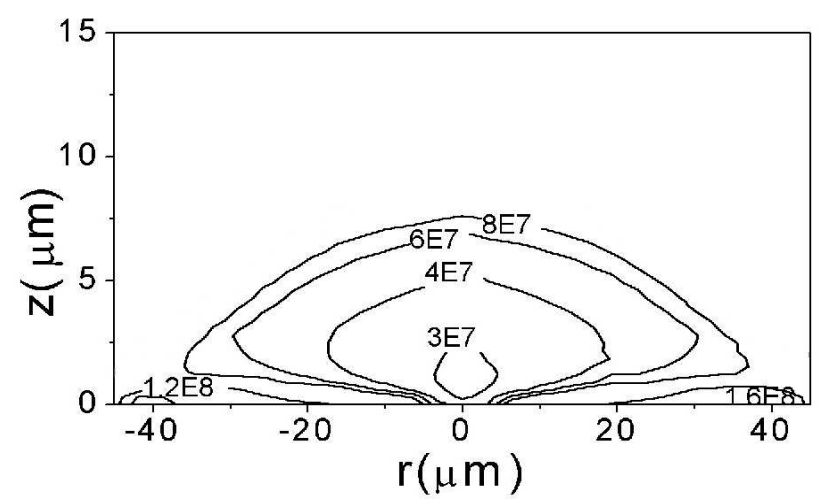

Fig.8 Profile of temperature gradient field during solidification: $G(\mathrm{~K} / \mathrm{m})$.

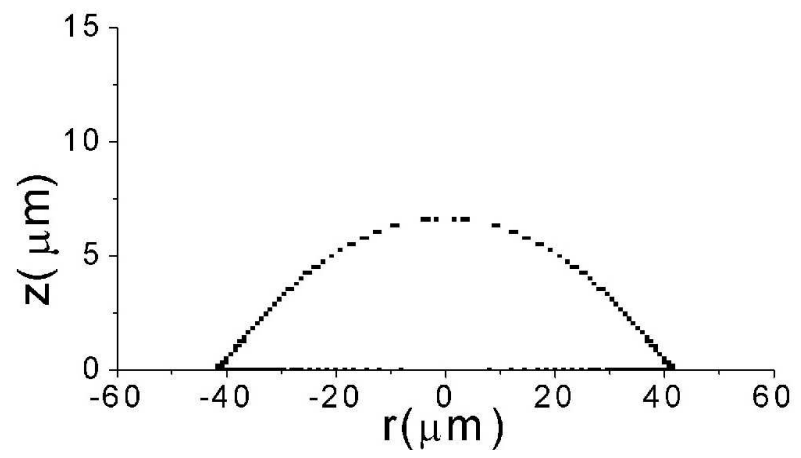

Fig.7 Shapes of molten zone for different values of $\varepsilon$ when central point begins to evaporate.

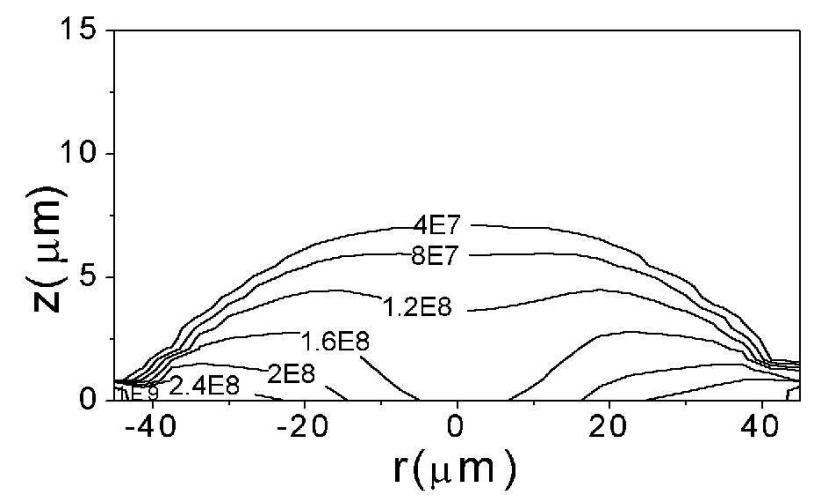

Fig.9 Profile of cooling velocity field during solidification: $V(\mathrm{~K} / \mathrm{s})$. 
Mushy Zone and Its Effect. We use $\varepsilon$ to denote the half width of mushy zone and $\varepsilon$ takes the value of $1.0 \mathrm{~K}, 5.0 \mathrm{~K}, 12.5 \mathrm{~K}, 15.0 \mathrm{~K}$ and $20.0 \mathrm{~K}$, respectively. From Fig.7, it is observed that the five pool shapes are nearly coincident, indicating the existence of mushy zone being of little effect on such a process. Since we assumed that there is no thermodynamic property difference in the three zones, the characteristics of mushy zone are rarely reflected.

Solidification. Figure 8 is the result showing the profile of magnitude contours for temperature gradient field, in which the value of temperature gradient $G$ at each point was derived from the corresponding moment of solidification. It is seen that in most parts of the molten pool the order of magnitude for $G$ is between $10^{7}$ to $10^{8} \mathrm{~K} / \mathrm{m}$. The regions with the highest value are the surface edge and the boundary between molten pool and the solid matrix. At some small local regions of surface edge, the value of $G$ is even so high as $10^{10} \mathrm{~K} / \mathrm{m}$. Figure 9 is the result showing the profile of magnitude contours for the average cooling rate during solidification. It is seen that the cooling rate $V$ is between $10^{7}$ and $10^{8} \mathrm{~K} / \mathrm{s}$. The region with the highest value of $V$ is the surface edge. Figures 8 and 9 indicate that during the solidification process, very high temperature gradient and cooling rate prevail. These are typical characteristics of pulsed laser treatment and will lead to the formation of very fine microstructure or possibly the formation of amorphous domains.

\section{Conclusions}

The evolution of temperature field for the surface layer of stainless steel due to pulsed Nd:YAG laser irradiation is numerically investigated. A 2-D axisymmetrical model with moving boundary is established and the enthalpy method was effectively applied to solve the problem. The following conclusions are drawn from the calculation results.

(1) During the melting process $(\sim 10 \mu \mathrm{s})$, the molten pool develops faster in the depth direction than in the radial direction. The existence of latent heat makes the molten pool become shallow and delays the occurrence of vaporization.

(2) At the start point of solidification, the temperature gradient is as high as $10^{7}$ to $10^{8} \mathrm{~K} / \mathrm{m}$. During the solidification process, the cooling rate is as high as $10^{7}$ and $10^{8} \mathrm{~K} / \mathrm{s}$. Such an extreme condition will lead to the formation of matastable microstructure of metallic materials.

Acknowledgements: This paper was supported by the National Natural Science Foundation of China and the Chinese Academy of Sciences.

\section{References}

[1] Mingjiang Yang: Research and applications of Nd:YAG laser-texturing equipment, science and technology appraisal report, Chinese Academy of Sciences, Beijing, 1993 (In Chinese)

[2] Guangnan Chen: Applied Laser, 16 (1996), p. 155 (In Chinese)

[3] W.F. Lucas: Differential Equation Models (Springer-verlag Inc, New York 1983)

[4] J. Crank: Free and Moving Boundary Problems (Clarendon Press, Oxford 1984)

[5] J. Ockendon and W.R. Hodgkins: (eds.) Moving Boundary Problems in Heat Flow and Diffusion (Clarendon Press, Oxford 1975)

[6] A.B. Crowley: Int. J. Heat Mass Transfer, 21 (1978), p.215

[7] A.A. Rostami, R. Greif and R.E. Russo: Int. J. Heat Mass Transfer, 35 (1992), p.2161

[8] V.R. Voller: Appl. Math. Modelling, 11 (1987), p.110

[9] J.L. Ma (ed.): Practical Thermal Engineering Handbook (Hydraulic \& Electric Engineering Press, Beijing 1988), (In Chinese)

[10]C.L. Chan and J. Mazumder: J. Appl. Phys., 62 (1987), p.4579 
Progress in Experimental and Computational Mechanics in Engineering

10.4028/www.scientific.net/KEM.243-244

Numerical Simulation of Temperature Field Evolution of Metallic Alloy Surface due to Pulsed Laser Treatment

10.4028/www.scientific.net/KEM.243-244.481

\section{DOI References}

[6] A.B. Crowley: Int. J. Heat Mass Transfer, 21 (1978), p.215

doi:10.1016/0017-9310(78)90225-9

[7] A.A. Rostami, R. Greif and R.E. Russo: Int. J. Heat Mass Transfer, 35 (1992), p.2161

doi:10.1016/0017-9310(92)90060-6

[8] V.R. Voller: Appl. Math. Modelling, 11 (1987), p.110

doi:10.1016/0307-904X(87)90154-5 